\title{
Hadronic contribution of light by light scattering in the energy spectrum of muonic hydrogen
}

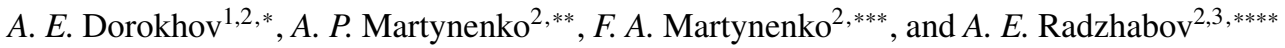 \\ ${ }^{1}$ Joint Institute for Nuclear Research, BLTP, 141980, Moscow region, Dubna, Russia \\ ${ }^{2}$ Samara University, 443086, Samara, Russia \\ ${ }^{3}$ Matrosov Institute for System Dynamics and Control Theory SB RAS, 664033, Irkutsk, Russia
}

\begin{abstract}
The contribution from scalar meson (S) exchange to the potential of the muon-proton interaction in muonic hydrogen induced by the scalar meson coupling to the two photon state is calculated. An estimate of transition form factor $S \rightarrow \gamma \gamma$ is given on the basis of the quark model and experimental data on the decay widths $\Gamma_{S \gamma \gamma}$. It is shown that scalar meson exchange contribution to the Lamb shift in muonic hydrogen $\Delta E^{L s}(2 P-2 S)$ is large and important for a comparison with precise experimental data.
\end{abstract}

\section{Introduction}

Among various electromagnetic interactions, the processes of two-photon meson production take a special place. First, they have been studied experimentally for quite a long period of time, for which a rich material has been accumulated [1]. Secondly, with the development of the quark model and nonperturbative methods of quantum chromodynamics, these reactions, as well as the reverse decay processes of mesons into two photons, were constantly in the field of intensive theoretical studies.

A new round of interest in $\gamma+\gamma \rightarrow$ meson processes is connected with their possible role as a new source of interactions between leptons and nucleons. Since in atomic physics there are precise experiments to measure the fine and hyperfine structure of the spectrum, any new contributions to the particle interaction operator are of interest and can be studied experimentally. The first estimates of the contribution of effective meson exchanges in muonic hydrogen, which have already appeared, show that this contribution can be significant [2-7].

In our previous papers $[6,7]$ we have investigated the role of effective exchanges of axial vector and pseudoscalar mesons for the position of the energy levels in muonic hydrogen. In this study we extend our analysis to the case of scalar mesons. There are several scalar mesons with the mass near $1 \mathrm{GeV}$, which can contribute to the effective muon-proton interaction: $\sigma(550), f_{0}(980), a_{0}(980), f_{0}(1370)$. So, the numerical calculation of the corresponding shifts in the fine and hyperfine structure of muonic atoms looks as an important task which can shed light on the proton charge radius puzzle.

\footnotetext{
*e-mail: dorokhov@theor.jinr.ru

**e-mail: a.p.martynenko@samsu.ru

***e-mail: f.a.martynenko@gmail.com

****e-mail: aradzh@icc.ru
} 


\section{General formalism}

The amplitude of the one-meson exchange between the muon and the proton arises as a result of the transition of two virtual photons into a scalar meson. The vertex function describing this process plays a central role in the study of meson exchange, since a good prediction of the magnitude of the shift in energy levels depends primarily on it. The general parameterization of scalar meson - two photon vertex function takes the following form [8, 9]:

$$
\begin{gathered}
T_{S}^{\mu v}\left(t, k_{1}, k_{2}\right)=4 \pi \alpha\left\{A\left(t^{2}, k_{1}^{2}, k_{2}^{2}\right)\left(g^{\mu v}\left(k_{1} \cdot k_{2}\right)-k_{1}^{v} k_{2}^{\mu}\right)+\right. \\
\left.B\left(t^{2}, k_{1}^{2}, k_{2}^{2}\right)\left(k_{2}^{\mu} k_{1}^{2}-k_{1}^{\mu}\left(k_{1} \cdot k_{2}\right)\right)\left(k_{1}^{v} k_{2}^{2}-k_{2}^{v}\left(k_{1} \cdot k_{2}\right)\right)\right\},
\end{gathered}
$$

where $A\left(t^{2}, k_{1}^{2}, k_{2}^{2}\right), B\left(t^{2}, k_{1}^{2}, k_{2}^{2}\right)$ are two scalar functions on three variables, $k_{1,2}$ are four momenta of virtual photons, $t$ is the four momentum of scalar meson. Then the muon-proton interaction amplitude via the scalar meson exchange can be written as follows:

$$
i \mathcal{M}=\frac{\alpha^{2} g_{s}}{\pi^{2}} \int \frac{d^{4} k A\left(t^{2}, k^{2}, k^{2}\right)\left(g^{\mu v}\left(k_{1} \cdot k_{2}\right)-k_{1}^{v} k_{2}^{\mu}\right)\left[\bar{u}\left(q_{1}\right) \gamma_{\mu}\left(\hat{p}_{1}-\hat{k}+m_{1}\right) \gamma_{v} u\left(p_{1}\right)\right]\left[\bar{v}\left(p_{2}\right) v\left(q_{2}\right)\right]}{k^{4}\left(k^{2}-2 m_{1} k_{0}\right)\left(\mathbf{t}^{2}+M_{s}^{2}\right)},
$$

where $p_{1}, p_{2}$ are four momenta of particles in the initial state, $q_{1}, q_{2}$ are four momenta of particles in final state. We set $t=q_{1}-p_{1}=0$ because this momentum is small and we consider further the leading order contribution to the interaction operator in the fine structure constant $\alpha$. This leads to the cancellation of the term with the function $B\left(p^{2}, k_{1}^{2}, k_{2}^{2}\right)$. Using projection operator on muon-proton states with spin $S=0, S=1$ [10] we can construct the interaction operator for these states. For example, in case of the triplet state we find:

$$
\begin{gathered}
i \mathcal{M}\left({ }^{3} S_{1}\right)=\frac{\alpha^{2} g_{s}}{16 m_{1}^{2} m_{2}^{2} \pi^{2}} \int \frac{d^{4} k}{k^{4}\left(k^{2}-2 k_{0} m_{1}\right)} A\left(t^{2}, k^{2}, k^{2}\right)\left(g_{\mu v} k^{2}-k_{\mu} k_{v}\right) \\
\operatorname{Tr}\left[\frac{1+\gamma_{0}}{2 \sqrt{2}} \hat{\varepsilon}\left(\hat{p}_{2}-m_{2}\right)\left(\hat{q}_{2}-m_{2}\right) \hat{\varepsilon}^{*} \frac{1+\gamma_{0}}{2 \sqrt{2}}\left(\hat{q}_{1}+m_{1}\right) \gamma_{\mu}\left(\hat{p}_{1}-\hat{k}+m_{1}\right) \gamma_{\nu}\left(\hat{p}_{1}+m_{1}\right)\right] \frac{1}{\mathbf{t}^{2}+M_{s}^{2}},
\end{gathered}
$$

where $m_{1}, m_{2}$ are the masses of a muon and proton, $M_{S}$ is the mass of scalar meson. After the trace calculation using the package Form we obtain the following in the leading order:

$$
\mathcal{T}_{2 S}=k^{2}\left(3 k_{0}+2 m_{1}\right)-2 m_{1} k_{0}^{2}
$$

The same contribution takes place for the singlet state ${ }^{1} S_{0}$, so in the leading order the scalar meson exchange does not contribute to the hyperfine structure. At the same time there is a shift of the level $2 S$ as the whole which is determined by (4). The typical momentum integral contributing to the shift has the following form:

$$
\mathcal{I}_{1}=\int \frac{d^{4} k\left(k^{2}+2 k_{0}^{2}\right)}{k^{2}\left(k^{4}+a_{1}^{2} k_{0}^{2}\right)} \frac{1}{\left(k^{2}+1\right)^{2}}=\frac{\pi^{2}}{12}\left[-9+36 \ln 2+2 a_{1}^{2}(-7+12 \ln 2)-12\left(3+2 a_{1}^{2}\right) \ln a_{1}\right],
$$

here $k$ is a dimensionless variable and $a_{1}=2 m_{1} / \Lambda$. The analytical value $\mathcal{I}_{1}$ is presented after expansion over $2 m_{1} / \Lambda$ up to the terms of the second order. This integral appears if we suppose that the parameterization of function $A\left(t^{2}, k^{2}, k^{2}\right)$ for scalar mesons has the same form as for pseudoscalar and axial-vector mesons (monopole form for variables $k_{1}$ and $k_{2}$ ):

$$
A\left(t^{2}, k^{2}, k^{2}\right)=A_{S} \frac{\Lambda^{4}}{\left(\Lambda^{2}+k^{2}\right)^{2}},
$$


where $A_{S}=A(0,0,0)$. The parameterization of the form (6) is widely used in the analysis of different experimental data [11]. The contribution to the interaction potential takes the form:

$$
\Delta V_{2 S}^{L s}(t)=\frac{\alpha^{2} g_{s} m_{1} A_{S}}{6}\left[-9+36 \ln 2+2 a_{1}^{2}(-7+12 \ln 2)-12\left(3+2 a_{1}^{2}\right) \ln a_{1}\right] \frac{1}{\mathbf{t}^{2}+M_{s}^{2}} .
$$

Averaging (7) over the wave function of $2 S$-state we obtain the shift of $2 S$-level in the form:

$$
\Delta E^{L s}(2 S)=\frac{\alpha^{5} \mu^{3} g_{s} m_{1} A_{S}}{96 \pi M_{S}^{2}} \frac{\left(2+\frac{W^{2}}{M_{S}^{2}}\right)}{\left(1+\frac{W}{M_{S}}\right)^{4}}\left[-9+36 \ln 2+2 a_{1}^{2}(-7+12 \ln 2)-12\left(3+2 a_{1}^{2}\right) \ln a_{1}\right] .
$$

The analytical expression (8) is used below for a numerical estimate of the contribution. To do this, you need to have reliable parameter values, including $A_{S}$.

To construct the particle interaction operator in the case of $2 P$-state, we use again the tensor method based on the tensor representation of wave functions and projection operators for $P$-states of muonic hydrogen [12]. It is convenient to work in the momentum representation, where the $2 P$-state wave function of the muonic hydrogen atom has the form:

$$
\psi_{2 P}(\mathbf{p})=\left(\varepsilon \cdot n_{p}\right) R_{21}(p),
$$

where $\varepsilon_{\delta}(L)$ is the polarization vector of orbital motion with the normalization condition $\varepsilon^{2}=3 / 4 \pi, n_{p}=(0, \mathbf{p} / p), R_{21}(p)$ is the radial wave function in momentum representation. At the first step of the calculation we project the muon on the state with angular momentum $j=1 / 2$ by means of the following projection operator [13]:

$$
\hat{\Pi}_{\omega}=\left[\varepsilon_{\omega}(0) u(0)\right]_{j=1 / 2}=\frac{1}{\sqrt{3}} \gamma_{5}\left(\gamma_{\omega}-v_{\omega}\right) \psi(0),
$$

where the field operator $\psi(0)$ describes the muon state with momentum $j=1 / 2$ in the rest frame. Then the muon-proton interaction amplitude in $P$-state due to scalar meson exchange has the following general structure:

$$
\begin{gathered}
i \mathcal{M}=\frac{\alpha^{2} g_{s}}{16 m_{1}^{2} m_{2}^{2} \pi^{2}} \int \frac{d^{4} k}{\left.k^{(} t-k\right)^{2}} \frac{1}{\left.k^{2}-2 m_{1} k_{0}\right)} A\left(t^{2}, k^{2},(t-k)^{2}\right)\left(g_{\mu \nu} k(t-k)-k_{\mu}(t-k)_{v}\right) \times \\
\frac{1}{3}\left[\bar{\psi}(0)\left(\gamma_{\omega}-v_{\omega}\right) \gamma_{5}\left(\hat{q}_{1}+m_{1}\right) \gamma_{\mu}\left(\hat{p}_{1}-\hat{k}+m_{1}\right) \gamma_{v}\left(\hat{p}_{1}+m_{1}\right) \gamma_{5}\left(\gamma_{\lambda}-v_{\lambda}\right) \psi(0)\right] \times \\
{\left[\bar{v}(0)\left(\hat{p}_{2}-m_{2}\right)\left(\hat{q}_{2}-m_{2}\right) v(0)\right] n_{p}^{\omega} n_{q}^{\lambda} \frac{1}{\mathbf{t}^{2}+M_{s}^{2}} .}
\end{gathered}
$$

At the second step we introduce projection operators on the states with the total angular momentum $\mathrm{F}=1$ and $\mathrm{F}=0$. This allows one to estimate the hyperfine structure of $2 P_{1 / 2}$ energy level. As it follows from the calculation, the scalar meson exchange has no contribution to the hyperfine structure in the leading order in $\alpha$. But the shifts of states $2^{3} P_{1 / 2}$ and $2^{1} P_{1 / 2}$ take place. The corresponding potential can be written in the form:

$$
\begin{gathered}
\Delta V_{2 P}^{L s}(\mathbf{t})=\frac{\alpha^{2} g_{s}}{16 m_{1}^{2} m_{2}^{2} \pi^{2}} \int \frac{d^{4} k}{\left.k^{(} t-k\right)^{2}} \frac{1}{\left.k^{2}-2 m_{1} k_{0}\right)} A\left(t^{2}, k^{2},(t-k)^{2}\right)\left(g_{\mu v} k(t-k)-k_{\mu}(t-k)_{v}\right) \times \\
\frac{1}{72} \operatorname{Tr}\left[\gamma_{\alpha}(1+\hat{v})\left(\gamma_{\omega}-v_{\omega}\right) \gamma_{5}\left(\hat{q}_{1}+m_{1}\right) \gamma_{\mu}\left(\hat{p}_{1}-\hat{k}+m_{1}\right) \gamma_{\nu}\left(\hat{p}_{1}+m_{1}\right) \gamma_{5}\left(\gamma_{\lambda}-v_{\lambda}\right)(1+\hat{v}) \gamma_{\beta} \times\right.
\end{gathered}
$$




$$
\left.\left(\hat{p}_{2}-m_{2}\right)\left(\hat{q}_{2}-m_{2}\right)\right]\left(-g_{\alpha \beta}+v_{\alpha} v_{\beta}\right) n_{p}^{\omega} n_{q}^{\lambda} \frac{1}{\mathbf{t}^{2}+M_{s}^{2}} .
$$

Simplifications in (12) using Form lead to the following expression in the leading order:

$$
\mathcal{T}_{2 P}=\frac{(\mathbf{p q})}{p q}\left[-\frac{2}{3} m_{1} k_{0}^{2}+k^{2} k_{0}+\frac{2}{3} m_{1} k^{2}\right]+p q\left(-\frac{5}{18} \frac{k_{0}^{2}}{m_{1}}+\frac{1}{4} \frac{k_{0} k^{2}}{m_{1}^{2}}-\frac{1}{18} \frac{k^{2}}{m_{1}}\right) .
$$

Taking into account that $|\mathbf{p}| \sim \mu \alpha,|\mathbf{q}| \sim \mu \alpha$ we can see that the fist term in (13) has the leading order. Nevertheless, in calculating the integral over the angles in the matrix element, the order of these two terms is equalized. On the whole, it turns out that expression (13) gives a correction of the order of $\alpha^{7} m_{1}^{2} / M_{S}^{2}$. The integration in $\mathrm{p}$ and $\mathrm{q}$ with radial wave functions $R_{21}(p)$ can be done analytically:

$$
\begin{gathered}
\mathcal{J}_{2 P}^{(1)}=\int R_{21}(q) \frac{d \mathbf{q}}{(2 \pi)^{3 / 2}} \frac{\frac{\mathbf{p q}}{p q}}{(\mathbf{p}-\mathbf{q})^{2}+M_{s}^{2}} R_{21}(p) \frac{d \mathbf{p}}{(2 \pi)^{3 / 2}}=\frac{W^{5}}{4 M_{s}^{4}} \frac{1}{\left(1+\frac{W}{M_{s}}\right)^{4}}, \\
\mathcal{J}_{2 P}^{(2)}=\int R_{21}(q) \frac{d \mathbf{q}}{(2 \pi)^{3 / 2}} \frac{\frac{p q}{m_{1}^{2}}}{(\mathbf{p}-\mathbf{q})^{2}+M_{s}^{2}} R_{21}(p) \frac{d \mathbf{p}}{(2 \pi)^{3 / 2}}=\frac{W^{5}}{8 M_{s}^{2} m_{1}^{2}} \frac{3+4 \frac{W}{M_{S}}+\frac{3 W^{2}}{2 M_{S}^{2}}}{\left(1+\frac{W}{M_{s}}\right)^{4}} .
\end{gathered}
$$

Another type integrals are connected with the loop variable k. One of integrals with the first function from (13) is calculated in (5). The second function from (13) gives the integral:

$$
\mathcal{I}_{2}=\frac{m_{1}}{18} \int \frac{d^{4} k\left(4 k_{0}^{2}-k^{2}\right)}{k^{2}\left(k^{4}+4 m_{1}^{2} k_{0}^{2}\right)} \frac{\Lambda^{4}}{\left(\Lambda^{2}+k^{2}\right)^{2}}=\frac{\pi^{2}}{108} m_{1}\left[-9+a_{1}^{2}(-5+6 \ln 2)-6 a_{1}^{2} \ln a_{1}\right],
$$

which is presented after expansion over $2 m_{1} / \Lambda$ up to terms of the second order. Taking together intermediate relations we obtain the shift of $2 P$-state in the form:

$$
\begin{aligned}
\Delta E^{L s}(2 P)= & \frac{\alpha^{7} \mu^{5} g_{s} A_{S}}{288 \pi m_{1} M_{s}^{2}\left(1+\frac{W}{M_{s}}\right)^{4}}\left\{\left[\frac{3}{4}+\frac{W}{M_{S}}+\frac{3}{8} \frac{W^{2}}{M_{S}^{2}}\right]\left[-9+a_{1}^{2}(-5+6 \ln 2)-6 a_{1}^{2} \ln a_{1}\right]-\right. \\
& \left.\frac{3 m_{1}^{2}}{M_{S}^{2}}\left[-9+36 \ln 2+2 a_{1}^{2}(-7+12 \ln 2)-12\left(3+2 a_{1}^{2}\right) \ln a_{1}\right]\right\}
\end{aligned}
$$

Two expressions (8), (17) give contributions which are different in sign just as in the case of pseudoscalar meson. They contribute to the Lamb shift $(2 P-2 S)$. Numerical values of (8), (17) are obtained below on the basis of quark model.

\section{Transition form factor $2 \gamma \rightarrow S$}

One of the main quantities that determine the energy shifts (8), (17), is the vertex function, in which two virtual photons are transformed into a scalar meson. In the local quark model it is given by the loop integral of the following form:

$$
T_{S}^{\mu \nu}=4 \pi \alpha \int \frac{d^{4} k}{(2 \pi)^{4}} \operatorname{Tr}\left[\gamma^{\mu} \frac{\left(\hat{k}+m_{q}\right)}{\left(k^{2}-m_{q}^{2}\right)} \gamma^{\nu} \frac{\left(\hat{k}-\hat{k}_{2}+m_{q}\right)}{\left[\left(k-k_{2}\right)^{2}-m_{q}^{2}\right]} \frac{\left(\hat{k}+\hat{k}_{1}+m_{q}\right)}{\left[\left(k+k_{1}\right)^{2}-m_{q}^{2}\right]}\right]+\left(k_{1}, \mu\right) \leftrightarrow\left(k_{2}, v\right) .
$$

As noted above, this tensor is determined by two scalar functions $A\left(t^{2}, k_{1}^{2}, k_{2}^{2}\right)$ and $B\left(t^{2}, k_{1}^{2}, k_{2}^{2}\right)$. In the kinematics $t^{2}=0, k_{1}^{2}=k_{2}^{2}=k^{2}$ of interest to us, only the contribution of the function $A\left(t^{2}, k_{1}^{2}, k_{2}^{2}\right)$ remains. In the local quark model, it has the form:

$$
A\left(t^{2}, k_{1}^{2}, k_{2}^{2}\right)=g_{S q q} \frac{N_{c}}{2 \pi^{2}} \operatorname{Tr}_{f}\left[\tau_{M} Q Q\right] I_{S \gamma \gamma}\left(t^{2}, k_{1}^{2}, k_{2}^{2}\right) .
$$


Table 1. Scalar mesons contribution to the energy spectrum of muonic hydrogen

\begin{tabular}{|c|c|c|c|c|c|}
\hline $\begin{array}{c}\text { Scalar } \\
\text { meson }\end{array}$ & $I^{G}\left(J^{P C}\right)$ & $\begin{array}{c}\Gamma_{\gamma \gamma}^{\text {xp }} \\
\text { in keV }\end{array}$ & $\begin{array}{c}\Lambda_{S} \\
\text { in MeV }\end{array}$ & $\begin{array}{c}A_{S} \\
\text { in } \mathrm{GeV}^{-1}\end{array}$ & $\begin{array}{c}\Delta E^{L s}(2 P-2 S) \\
\text { in } \mu e V\end{array}$ \\
\hline$\sigma(550)$ & $0^{+}\left(0^{++}\right)$ & 4.5 & 2000 & -0.299 & 11.2657 \\
\hline$f_{0}(980)$ & $0^{+}\left(0^{++}\right)$ & 0.33 & 2000 & -0.034 & 0.8651 \\
\hline$a_{0}(980)$ & $1^{-}\left(0^{++}\right)$ & 0.30 & 2000 & -0.032 & 0.8142 \\
\hline$f_{0}(1370)$ & $0^{+}\left(0^{++}\right)$ & 4.5 & 2000 & -0.075 & 1.3661 \\
\hline
\end{tabular}

For the isoscalar meson $(\sigma)$ the trace over flavour $\operatorname{Tr}_{f}\left[\tau_{M} Q Q\right]=5 / 9$, but for the isovector state $\left(a_{0}(980)\right) \operatorname{Tr}_{f}\left[\tau_{M} Q Q\right]=1 / 3$. The coupling constant of scalar $\sigma$-meson with the quarks $g_{\sigma q q}=\frac{m_{q}}{f_{\pi}}$, where $m_{q}$ is the mass of light quark and the pion decay constant $f_{\pi}=0.0924 \mathrm{GeV}$. In the case of other scalar mesons we can consider $g_{S q q}$ as additional parameter which can be fixed by means of experimental data on decay width $\Gamma_{S \gamma \gamma}^{\exp }$. In the local quark model the mass of scalar $\sigma$-meson is $M_{\sigma}^{2}=M_{\pi}^{2}+4 m_{q}^{2}$. The loop momentum integral $I_{S \gamma \gamma}\left(t^{2}, k_{1}^{2}, k_{2}^{2}\right)$ in (18) can be directly calculated using the Feynman parameterization and intermediate dimensional regularization. The result for it is finite and can be presented as follows:

$$
\begin{gathered}
I_{S \gamma \gamma}\left(t^{2}, k_{1}^{2}, k_{2}^{2}\right)=-\frac{m_{q}}{\left(k_{1} \cdot k_{2}\right)} \int_{0}^{1} d\left\{x_{1} x_{2} x_{3}\right\} \frac{B+\left(1-2 x_{1} x_{2}\right)\left(k_{1} \cdot k_{2}\right)+k_{1}^{2} x_{1}^{2}+k_{2}^{2} x_{2}^{2}}{B+m_{q}^{2}}, \\
d\left\{x_{1} x_{2} x_{3}\right\}=d\left(x_{1} x_{2} x_{3}\right) \delta\left[1-\left(x_{1}+x_{2}+x_{3}\right)\right], B=-\left(t^{2} x_{1} x_{2}+k_{1}^{2} x_{1} x_{3}+k_{2}^{2} x_{2} x_{3}\right) .
\end{gathered}
$$

Setting further $t^{2}=0, k_{1}^{2}=k_{2}^{2}=-k^{2}$ we obtain for the integral (20):

$$
I_{S \gamma \gamma}\left(0,-k^{2},-k^{2}\right)=\frac{m_{q}}{k^{2}}\left(-2+\frac{4 m_{q}^{2} \ln \left(\frac{\sqrt{k^{2}} \sqrt{4 m_{q}^{2}+k^{2}}+2 m_{q}^{2}+k^{2}}{2 m_{q}^{2}}\right)}{\sqrt{k^{2}\left(4 m_{q}^{2}+k^{2}\right)}}\right)
$$

The expansion (21) at small momenta $\mathrm{k}$ can be used to estimate parameter $A_{S}$. So, for the isoscalar and isovector cases we obtain:

$$
A_{S}^{I=0}=A(0,0,0)=-\frac{1}{2 \pi^{2} f_{\pi}} \frac{5}{9}, \quad A_{S}^{I=1}=A(0,0,0)=-\frac{1}{2 \pi^{2} f_{\pi}} \frac{1}{3} .
$$

It is useful to compare the results of calculating parameter $A_{S}$ in the local quark model with estimates of this quantity based on the experimental data on decay of scalar mesons into two photons. The calculation of $A_{S}=A\left(t^{2}=M_{s}^{2}, 0,0\right)$ can be carried out on the basis of the quark model. Using the quark-loop amplitude contributing to the decay $S \rightarrow \gamma+\gamma$ we can present the general structure of the two-photon scalar meson decay amplitudes as follows $[14,15]$ :

$$
T_{S \gamma \gamma}^{\mu \nu}=-\frac{\alpha g_{S u}}{\pi m_{u}}\left(g^{\mu \nu}\left(k_{1} k_{2}\right)-k_{1}^{\nu} k_{2}^{\mu}\right) a_{S \gamma \gamma} .
$$

Then the expression for the decay width which is measured in experiment $[14,16,17]$, has the following form:

$$
\Gamma_{S \gamma \gamma}=\frac{M_{s}^{3} \alpha^{2} g_{\sigma_{u}}^{2}}{64 \pi^{3} m_{u}^{2}}\left|a_{S \gamma \gamma}\right|^{2}
$$


Taking the experimental value of $\Gamma_{S \gamma \gamma}$ or its theoretical estimate we can find the value of phenomenological constant $a_{S \gamma \gamma}$ and relate it to our parameter $A_{S}$. The corresponding numerical values $A_{S}=g_{S u} a_{S \gamma \gamma} / 4 \pi^{2} m_{u} e$ for scalar mesons $\sigma(550), f_{0}(980), a_{0}(980), f_{0}(1370)$ are presented in Tab. 1 . To estimate $A_{S}(0,0,0)$ by the meson decay width, we introduce an additional factor outside the mass shell, based on the assumption that

$$
A_{S}(t, 0,0)=A_{S}\left(t=M_{S}^{2}, 0,0\right) e^{\frac{t-M_{S}^{2}}{M_{S}^{2}}} .
$$

In the local quark model different values of a parameter $A_{S}$ for scalar mesons are related with different values of coupling constants of scalar mesons with light quarks $g_{S u}$. It should be noted that the nature of many light scalar mesons has not been fully studied [18]. One of the widely discussed options is the idea of existence of an admixture of four-quark states in them. This circumstance introduces significant uncertainties in the calculation of the constant $A_{S}$ within the framework of theoretical models and, accordingly, in the calculation of the contribution to the Lamb shift $\Delta E^{L s}(2 P-2 S)$.

\section{Conclusion}

The numerical estimate of contributions of scalar mesons to the Lamb shift $(2 P-2 S)$ is shown in Tab. 1. It is obtained on the basis of equations (8), (17) taking the values of parameters for scalar mesons from $[5,14,16]$. Our result for $\sigma$-meson is in agreement with the previously obtained estimates in $[5,9]$. The obtained contributions of scalar mesons to the Lamb shift $(2 P-2 S)$ in muonic hydrogen are large and should be used for precise comparison with experimental data of CREMA collaboration [19, 20].

The work is supported by Russian Science Foundation (grant No. RSF 18-12-00128) and Russian Foundation for Basic Research (grant No. 18-32-00023) (F.A.M.).

\section{References}

[1] V. M. Budnev et al., Phys. Rep. 15, 181 (1975)

[2] F. Hagelstein, V. Pascalutsa, PoS CD 15, 077 (2016)

[3] H. Q. Zhou, H. R. Pang, Phys. Rev. A 92, 032512 (2015)

[4] N. T. Huong, E. Kou, B. Moussallam, Phys. Rev. D 9, 114005 (2016)

[5] H.-Q. Zhou, Phys. Rev. C 95, 025203 (2017)

[6] A. E. Dorokhov et al., Phys. Part. Nucl. Lett. 14, 857 (2017)

[7] A. E. Dorokhov et al., Phys. Lett. B 776, 105 (2018)

[8] A. E. Dorokhov, A. E. Radzhabov, and A. S. Zhevlakov, EPJ C 72, 2227 (2012)

[9] D. Borisyuk, Phys. Rev. C 96, 055201 (2017)

[10] A. P. Martynenko, F. A. Martynenko, and R. N. Faustov, JETP 124, 895 (2017)

[11] P. Achard et al. [L3 Collaboration], Phys. Lett. B 526, 269 (2002)

[12] R. N. Faustov et al., Phys. Rev. A 92, 052512 (2015)

[13] A. E. Dorokhov et al., Eur. Phys. Jour. A 54, 131 (2018)

[14] M. K. Volkov, E. A. Kuraev, and Yu. M. Bystritskiy, Phys. Atom. Nucl. 73, 443 (2010)

[15] F Giacosa, Th. Gutsche, and V. E. Lyubovitskij, Phys. Rev. D 77, 034007 (2008)

[16] C. Patrignani et al. (Particle Data Group), Chin. Phys. C 40, 100001 (2016)

[17] T. Branz, L. S. Geng, and E. Oset, Phys. Rev. D 81, 054037 (2010)

[18] N. N. Achasov and G. N. Shestakov, Phys. Usp. 54, 799 (2011)

[19] R. Pohl, A. Antognini, F. Nez et al., Nature 466, 213 (2010)

[20] A. Antognini et al., Science 339, 417 (2013) 